\title{
1 Models as games: a novel approach for 'gamesourcing' parameter data and 2 communicating complex models
}

3 Jeroen Minderman, A. Bradley Duthie, Isabel L. Jones, Laura Thomas-Walters, Adrian Bach,

4 Matthew Nuttall \& Nils Bunnefeld

5 Biological \& Environmental Sciences, University of Stirling, Stirling, FK9 4LA, UK

6 Corresponding author: jeroenminderman@gmail.com

\section{$7 \quad 1 \quad$ Summary}

8 1. Models have become indispensable tools in conservation science in the face of

3. We present players with a natural resource management game as a front-end to a socialecological modelling framework (Generalised Management Strategy Evaluation, GMSE). Players' actions replace a model algorithm making management decisions about a population of wild animals, which graze on crops and can thus lower agricultural yield. A number of non-player agents (farmers) respond through modelled algorithms to the player's management, taking actions that may affect their crop yield as well as the animal population. Players are asked to set their own management goal (e.g. maintain the animal 
population at a certain level or improve yield) and make decisions accordingly. Trial players were also asked to provide any feedback on both gameplay and purpose.

4. We demonstrate the utility of this approach by collecting and analysing game play data from a sample of trial plays, in which we systematically vary two model parameters, and allowing trial players to interact with the model through the game interface. As an illustration, we show how variations in land ownership and the number of farmers in the system affects decision-making patterns as well as population trajectories (extinction probabilities).

5. We discuss the potential and limitations of this model-game approach in the light of trial player feedback received. In particular, we highlight how a common concern about the game framework (perceived lack of "realism" or relevance to a specific context) are actually criticisms of the underlying model, as opposed to the game itself. This further highlights both the parallels between games and models, as well as the utility of modelgames to aid in communicating complex models. We conclude that videogames may be an effective tool for conservation and natural resource management, and that although they provide a promising means to collect data on human decision-making, it is vital to carefully consider both external validity and potential biases when doing so.

\section{$43 \quad 2 \quad$ Introduction}

44 In recent years, the use and application of models 1 has become widespread and indispensable in 45 conservation science, ranging from demonstrating the likely effects of climate change on 46 biodiversity (IPCC 2021) to supporting the understanding of fundamental processes in natural 47 resource management (e.g. Schlüter et al. 2012; Fryxell et al. 2010; Cusack et al. 2020). Given 48 the continued rapid global loss of biodiversity (Ceballos et al. 2015; Ceballos, Ehrlich, and Dirzo

1 We here use the term "model" to refer to any predictive quantitative model, although our focus is on predictive simulation models used for decision support. However, the arguments presented here equally apply to statistical models, particularly when used for prediction of trends. 
2017), understanding the mechanisms and consequences of such loss is vital for long-term sustainability. Although a number of drivers of biodiversity loss have been identified (e.g. Maxwell et al. 2016), one of the most prevalent and widespread is human exploitation of habitats and natural resources, both directly (e.g. through hunting or habitat loss to agriculture) or indirectly (e.g. through international trade in natural resources) (Wilting et al. 2017). Because resource use is fundamentally driven by economic and social processes, accurately predicting future changes is reliant as much on understanding human behaviour and decision-making (Milner-Gulland 2012; Schlüter et al. 2012) as it is on understanding resource dynamics themselves. Thus, the development of social-ecological models in which natural resource dynamics and human decision making interact is becoming increasingly urgent.

Cutting-edge modelling approaches have made significant progress towards this goal. For example, Orach, Duit, and Schlüter (2020) used an agent-based model to show how coalitions of interest groups can stabilise natural resource dynamics, whereas Cusack et al. (2020) used a novel agent-based modelling framework (Duthie et al. 2018) to assess the effect of lobbying on species extinction risk. Although such efforts represent significant progress in modelling complex social-ecological systems, their increased complexity poses two interlinked challenges. First, models are often difficult to communicate clearly to non-specialist audiences, and this challenge increases with model complexity (Grimm et al. 2006). This is particularly important for models of resource use in social-ecological systems, as they are often specifically intended for use by managers or stakeholders who may not have the required technical expertise. Much has been said about improving the uptake of models in such settings (e.g. Bunnefeld, Nicholson, and Milner-Gulland 2015; Addison et al. 2013; Schuwirth et al. 2019; Will et al. 2021), and 71 detailed documentation of the purpose, organisation and predictions has been highlighted as 72 particularly important (Grimm et al. 2020). Even so, frequently the evidence for practical uptake 73 of many models is limited (Addison et al. 2013; Bunnefeld, Nicholson, and Milner-Gulland 74 2015; Zasada et al. 2017). Second, their complexity implies the need for extensive data to 75 parameterise them effectively. In terms of social-ecological systems, while data to parameterise 76 the ecological component are often relatively easily available, the human decision-making 77 components are often based on limited theory and lack a general empirical basis (Groeneveld et 
al. 2017; Schwarz et al. 2020). Not only may this lead to limited predictive power, but stakeholders may also be unwilling to accept model results that they perceive as lacking an empirical basis (cf. model "quality" as in Kolkman et al. 2016). To maximise the adoption of complex social-ecological models as management tools, both appropriate representation of human decision-making, and effective communication, are therefore key.

Games have a long history of use in research (Sandbrook, Adams, and Monteferri 2015; Chabris 2017; Redpath et al. 2018), including as tools to aid the communication of complex ideas and processes to non-specialists (Garcia, Dray, and Waeber 2016; Tan et al. 2018), with recent work starting to leverage online and video games (Oultram 2013; Pérez and Guzmán-Duque 2014; Fjaellingsdal and Kloeckner 2019; Crowley, Silk, and Crowley). Given this long history, it is striking that the parallels between games, in particular videogames, and models are not recognised more widely. All models are abstract representations of environments, actors and relationships, with inputs (parameters) and outputs (predictions or inferences). Similarly, all games present a player with an environment in a given state (parameters), including one or more actors, who can take actions (inputs) to affect the environment for a given effect (outputs). It is worth stressing that every game has an underlying model that defines the state of the environment, relationships between objects in this environment, and inputs and outputs available to the player. However, while games are by definition designed with player interaction in mind, models rarely have user-facing or even user-friendly interfaces, and running or adapting them to specific circumstances usually relies on technical expertise. Casting models as games provides an opportunity to effectively improve the communication and understandability of even relatively complex models. Inputs and outputs may be presented in a visual way and adapted

100 depending on the type of audience, and both potential applications and limitations of the model can be demonstrated effectively.

102 In addition, presenting a model as a game provides an opportunity to empirically collect data on

103 how stakeholders make decisions in the modelled environment. Games have already been widely 104 used for data collection to answer specific questions (e.g. Meinzen-Dick et al. 2016; Villamor 105 and Badmos 2016; Rakotonarivo, Bell, et al. 2021; Rakotonarivo, Jones, et al. 2021) about what 
106 affects decision-making in social-ecological systems. Another potential application of presenting 107 model as games, which warrants further exploration, is using in-game decisions as a "big data" 108 source to improve the parameterisation of the underlying model itself. Many existing models 109 represent human decision-making by relatively crude algorithms (e.g. fully rational utility 110 maximisation) despite widespread recognition that this does not reflect real-world decision111 making (Groeneveld et al. 2017). By presenting real-world stakeholders with in-game decisions

112 that would otherwise be taken by a predefined algorithm, large data sets of actions and outcomes 113 may be collected. Given a large enough sample of players and in-game conditions, such data 114 might then be used to train decision-making algorithms that better reflect human decision115 making in natural resource management2. Although this "gamesourcing" or "Gamorithm" 116 (Sipper and Moore 2020) approach has already been widely used in a number of other fields 117 (from crowdsourcing accurate protein-structure models to classifying fluorescence microscopy 118 images, Khatib et al. 2011; Sullivan et al. 2018), it remains rare in conservation science (but see 119 van den Bergh et al. 2021). Thus, model-games can be considered "virtual laboratories" (Duthie 120 et al. 2021) to not only test specific hypotheses or predictions, but potentially also as an effective 121 method to source data to parameterise the underlying models based on in-game decisions by real 122 humans.

123 We aim to illustrate the potential for this model-game approach, both in terms of aiding model 124 communication as well data collection for improved parameterisation, by introducing 125 Animal\&Farm (A\&F). We developed $A \& F$ as a simple interactive game front-end for a complex 126 social-ecological modelling framework (GMSE), in which the player acts as the manager of a 127 virtual environment in which a population of wild grazing animals (the natural resource) may 128 adversely affect farming yield, with farmers acting to maximise their yield and potentially 129 hunting or deterring (through scaring) the animals. We argue that that by acting as an interface 130 between users (i.e. players) and complex underlying models with many components and

2 Note that there are limitations to this, and that data on decisions made would only be relevant to the context of the game; we discuss limitations in more detail below. 
131 assumptions, games can simultaneously (1) aid the communication and useability of the

132 underlying model and (2) can be used to gather data to improve the parameterisation of such

133 models. We first briefly summarise the underlying modelling framework, its potential and

134 limitations. Second, we describe both the structure of A\&F itself as well as its database back-

135 end. Third, we outline how this approach may be used to collect data on player decision-making

136 in simulated in silico experiments, and present some example results of doing so; noting that

137 these findings are intended as illustrative only. Finally, using test player feedback as a

138 foundation, we discuss both the limitations of this approach as well as its wider potential.

\section{Outline of approach}

140 A\&F is available to play online.

141 A\&F consists of two main components; (1) the underlying model(s) describing the wild grazing

142 animal ("resource") population dynamics, estimates of this population through an observation

143 process, and farmer actions, which are all implemented using the GMSE framework as described

144 below; and (2) the game interface for the underlying model, which allows the player to set

145 management actions (specifically, costs for farmer actions) that would otherwise be determined

146 by the management model in the default GMSE set up.

\section{$147 \quad 3.1 \quad$ Underlying model: GMSE}

148 We used GMSE to model the social-ecological system underlying A\&F. The GMSE R package

149 (https://cran.r-project.org/web/packages/GMSE/index.html) was designed as a flexible solution

150 for parameterising systems that model the management, observation, exploitation and population

151 dynamics of a natural resource (e.g. a population of hunted wildlife). In this section, we

152 summarise the basic functionality of GMSE as relevant to the present manuscript; for a full

153 description see Duthie et al. (2018) and Nilsson et al. (2021) (the latter containing an appendix

154 with the full ODD model description). 


\subsubsection{Basic introduction of GMSE principles and structures}

156 GMSE is an agent-based modelling framework consisting of four sequential submodels (Figure

157 1a) with three types of agents:

158 1. The resource model, consisting of $\square$ individual animal-agents (hereafter referred to as "animals") moving on a landscape of $\square \mathrm{x} \square$ cells.

1602 . The observation model which represents the process of observations (including a degree of uncertainty) of the animal population.

3. The manager model consists of a single agent (hereafter referred to as the "manager") which uses the observation of the animal population to make management decisions, affecting the permissiveness of actions for agents in the user model (below).

4. The user model, consisting of $\square$ individual agents, which in the current context represent land-owning farmers. Thus, we refer to these agents as "farmers" in the remainder of this manuscript, but note that we use the term "user model" to refer to the general submodel containing these agents to maintain consistency with the term used in the GMSE documentation (Duthie et al. 2018). Each farmer owns a given proportion of the landscape (this may or may not be an equal distribution, see below).

171 In each GMSE time step, both the manager and all farmers are allocated a (fixed) budget. In

172 GMSE terms, "budget" should be interpreted as a budget of general "resource"; conceptually this

173 may be interpreted as a financial budget, time, materials, or a combination thereof. Farmers may 174 allocate their budget to taking one of several potential actions on their land (here: hunting 175 animals, scaring animals off their land, or tending crops). Both former actions are common in the 176 management and control of grazing animals on croplands (e.g. grazing migratory wildfowl, 177 (Nilsson et al. 2016, 2021), with scaring for example including the use of acoustic deterrents. 
(a)

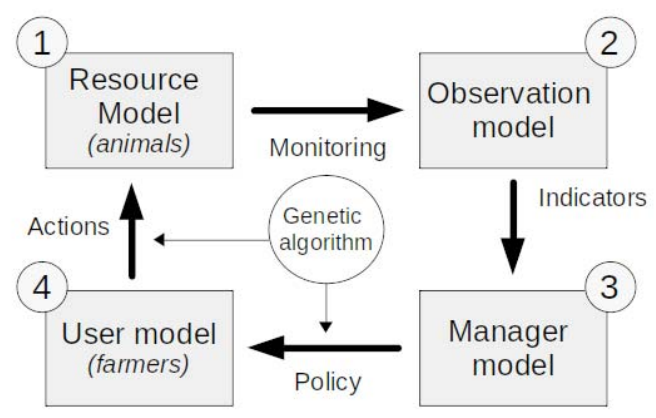

(b)

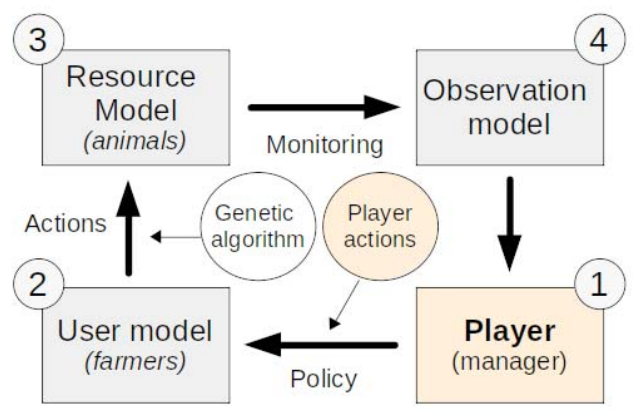

178

179 Figure 1. The basic structure of (a) the GMSE modelling framework and its default order of operations

180 with the genetic algorithm (GA) modelling the decision-making process of both users and manager, and

181 (b) the adaptation of the GMSE framework to accommodate the model-game approach presented here.

182 The grey boxes represent the various GMSE submodels, with the arrows representing the process links

183 between them. The yellow boxes and circles are the adapted components in the model-game adaptation,

184 with player interaction replacing the manager model in GMSE, and the underlying GA for the manager -

185 the GA is still used to make user decisions. Grey circles indicate the order of operations in each.

186 The goal for the manager is to maintain the animal population at a desired level (the management

187 target, normally set externally as a model parameter). The manager does so by controlling the

188 cost for farmer actions in the following time step. For example, a higher cost for hunting will

189 decrease the number of animals hunted by farmers leading to population growth, and a lower

190 cost for scaring will increase the number of farmers choosing scaring as an action.

191 Farmers aim to maximise agricultural yield from their land. By default, yield equals 1 per

192 landscape cell owned per time step, but this may be decreased by the presence of grazing animals

193 in a cell, and/or increased through tending crops. The rates of increase or decrease in yield

194 through grazing and tending crops respectively are controllable in GMSE but kept as constant

195 rates in the current A\&F implementation, see Duthie et al. (2018) and parameter references for

196 res_consume and tend_crop_yld in the gmse() function here: https://cran.r-

197 project.org/web/packages/GMSE/GMSE.pdf. Thus, although their objective does not directly

198 relate to the animals, farmers have an incentive to control the number of animals on their land to 
199 minimise potential negative effects on their yield. They can do this by allocating budget to 200 hunting or scaring animals. The former reduces the number of animals present in the landscape, 201 while the latter has a certain probability of moving an animal away from the farmers' land, for 202 the duration of the time step. The relative expected efficacy of the three possible actions 203 (hunting, scaring or tending crops) depends on the number of animals on their land, and the cost 204 of hunting and scaring set by the manager. Farmers can only take actions on land that they own.

205 By default, costs for farmer actions as set by the manager and actions taken by the farmers are 206 chosen using a genetic algorithm (GA), a heuristic optimisation algorithm that models the choice 207 of decision by mimicking the process of evolution by natural selection: a large number of 208 possible decisions are iteratively compared by assessing their outcome, with the decision that 209 maximises a given utility function (yield for farmers, and minimising distance to population 210 target for the manager, see Duthie et al. 2018) identified as the "fittest" (Hamblin 2013). The GA 211 is run separately for each agent (manager and each user) in each time step.

212 In the default resource (animal) model in GMSE, the animal population is modelled with a form 213 of logistic growth, with a small amount of random mortality added per time step and death 214 caused by hunting; for more detail see below and Duthie et al. (2018). In each time step, each 215 animal moves a given distance in a random direction, and feeds from the cell in which it is 216 present. In the current model, neither movement nor population growth rate is affected by 217 agricultural yield.

218 It is worthwhile stressing that in the current GMSE implementation, using the GA, both agent 219 types (farmers and the manager) have only a single goal they each aim for. Farmers aim to 220 maximise their yield, whereas the manager aims to minimise deviation from a given population 221 target - neither can balance multiple competing objectives. This is unlikely to be reflective of real 222 conservation scenarios, where it is common for conservation managers to at least recognise other 223 aims, if not take these explicitly into account when setting policy, and other stakeholders in the 224 system (e.g. farmers) commonly having some interest in conservation objectives (Redpath et al. 225 2017; Bunnefeld, Nicholson, and Milner-Gulland 2015). Human decision-making in such 226 scenarios is inevitably about balancing these different objectives, but parameterising algorithms 
227 that mimic such processes without reference to empirical data is very challenging (Constantino et 228 al. 2021; Dobson et al. 2019). Addressing this issue was a key motivation for the development of 229 the model-game approach presented here.

\subsection{Animal \& Farm}

\subsubsection{Structure as relating to GMSE}

232 In the default implementation of GMSE v0.7.0.0, a single time $\square$ step consists of a call to the 233 resource model, observation model, management model and user model, in that specific order; in 234 other words, a time step ends after farmer actions have been chosen (by the GA) and 235 implemented (Figure 1a). To allow players to assess the environment and interactively choose 236 management actions, A\&F uses a modified version of GMSE implemented in R version 4.1.1 237 (2021-08-10), the code for which is freely available: 238 https://github.com/ConFooBio/gmse/tree/man_control. In this version, the management model is 239 replaced by player inputs, and the order of operations is altered to accommodate this. Further 240 details are given in S1.

241 The current GMSE parameter values used by A\&F largely reflect default parameter values in 242 GMSE. This is a purely pragmatic choice: because we are not modelling a specific system here, 243 and instead aim only to illustrate the use of the A\&F platform in general terms, the specific 244 parameter values given below and in Table S2 should be interpreted as examples: we emphasise 245 that all these parameters are expected to be modified as appropriate for specific GMSE and A\&F 246 applications.

247 The example parameterisation used here simulates a landscape of 100x100 cells, divided into 248 farms owned by 4-12 farmers (the precise number and land distribution is randomly varied per 249 session, see 4.2 below). Farmers can take three possible actions: tending crops, hunting (culling) 250 animals, or scaring animals off their land. All submodels used in A\&F are currently the default 251 GMSE models (see S1), with the exception of the management model in time steps $\square>5$ where 252 the player assumes control over the management decisions (see below). We only give brief 253 details on GMSE itself here, for full details and descriptions of all models, see Duthie et al. 
254 (2018) and Nilsson et al. (2021). The animal population model uses the logistic growth form 255 with $\square_{\square}=1000, \square=0.3$ and $\square=5000$, meaning that in the absence of any management the 256 population will increase from the initial population size (1000) to carrying capacity (5000). The 257 observation model uses the default GMSE model (density-based sampling of a subset of the 258 environment); the manager can only base decisions on the observed number of animals (and thus 259 population trajectory plots in the game interface reflect observations only, which are subject to 260 an unknown - to the player - level of uncertainty). Both the management model (in the 261 initialisation steps) and user model use the genetic algorithm with default parameter settings. 262 Farmer budgets are set to 1500 units per time step, manager budgets to 1000 units (both for the 263 initial 5 time steps and the subsequent game play; see 3.1 .1 above for notes on the 264 conceptualisation of "budget"). Farmers aim to maximise yield from their land; their annual 265 budget is reset each year and is unaffected by yield. Yield is positively affected by tending crops 266 and may be negatively affected by the presence of grazing wild animals - thus hunting or scaring 267 may offset any potentially negative effects on yield.

268 Each subsequent A\&F time step consists of (1) player input, taking the place of the default 269 management model, in which the player can assess the environment using outputs provided (see 270 below) and choose management actions (costs for farmer actions), and (2) a modified GMSE 271 time step (following player confirmation of their inputs), including sequential calls to the default 272 user, resource and observation models (gmse_apply_UROM( )) (Figure 1b).

\section{$273 \quad 3.2 .2 \quad$ Player interface}

274 The player interface for A\&F is a web application coded in R, using Shiny (1.6.0), and packages 275 shinyjs (2.0.0), shinyBS (0.61), and waiter (0.2.2).

276 On starting a new game session, the player is presented with a series of introductory screens 277 explaining the background, flow, and objective of the game, after which they are asked to enter a 278 player name, which is stored and used to show player scores as the end of a session, compared to 279 previous sessions by other players. 


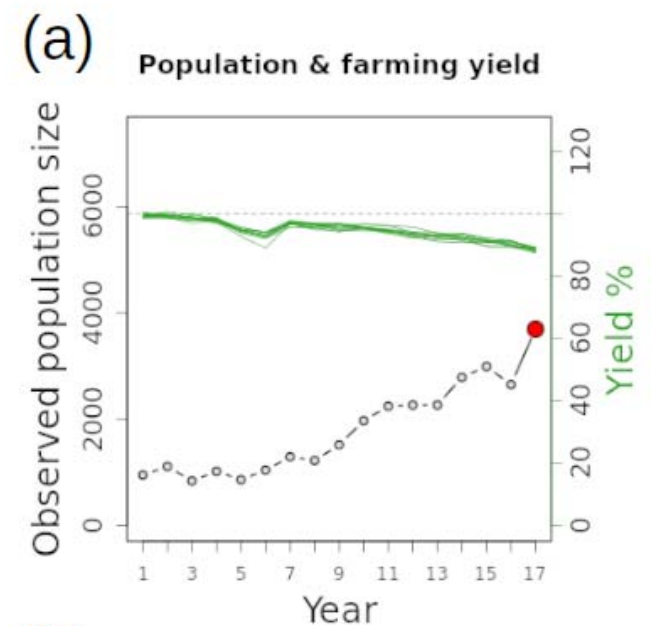

(b)

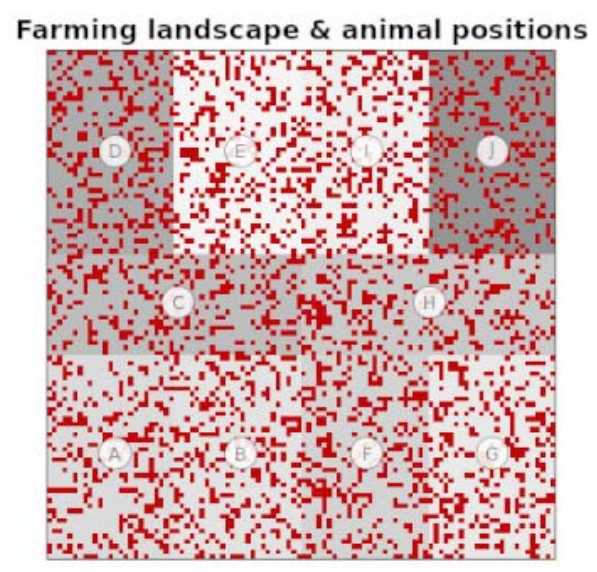

(d)
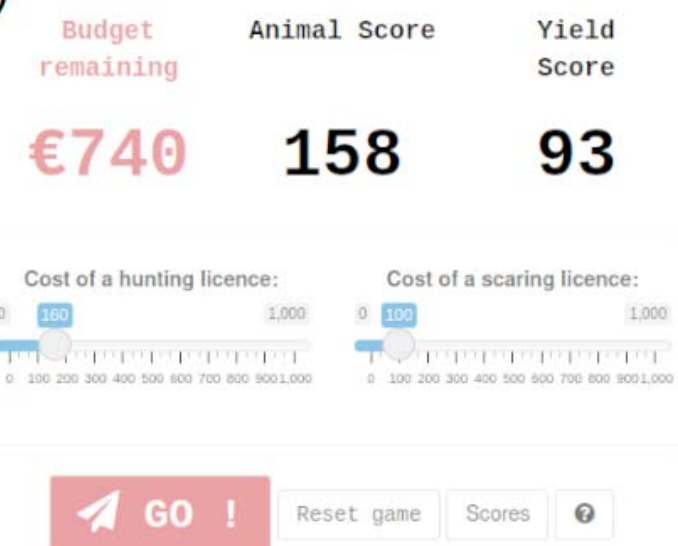

(c)

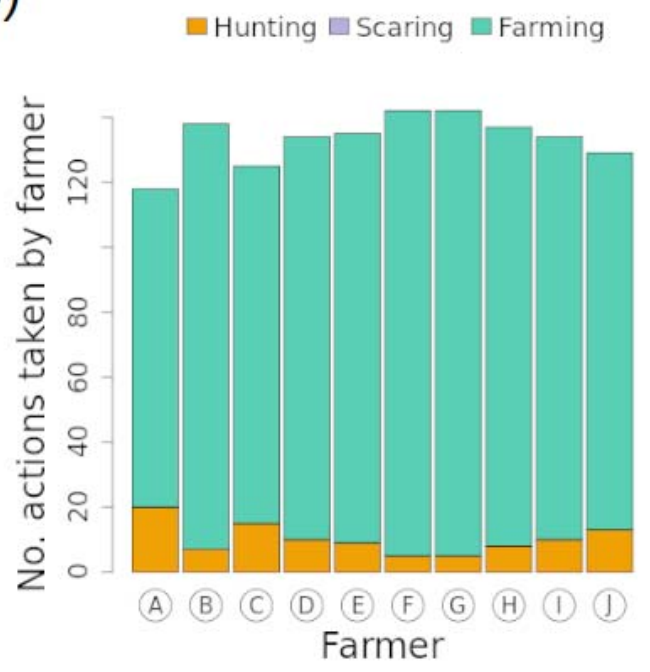

281 Figure 2. The 'Animal and Farm' main game interface, showing (a) the animal (resource) population

282 trajectory and yield per farmer, (b) the farming landscape with animal positions as red dots and farm

283 ownership indicated by the grey shades, (c) actions taken by farmers in the previous game round, and (d)

284 player inputs including a budget report and costs set for actions.

285 The main game screen consists of four components (Figure 2). First, a trajectory plot (Figure 2a)

286 showing (1) observed animal population numbers and (2) agricultural yield for each farmer in 287 each time step, up to time $\square$ (at the start of the game, this will show five observations from the 288 initialisation steps described above). Agricultural yield is expressed as a \% of "maximum 
unaffected yield," i.e. yield in the absence of damage from wildlife or investment in tending crops. Second, a plot of the landscape (Figure 2b) showing the distribution of farm ownership as

291 well as the position of animals at time $\square$. Third, a bar plot of the number of actions taken by each

292 farmer at time $\square$ (Figure 2c). Fourth, a report of the current management budget available (not

293 allocated), player scores (see 3.2.3 below), and player inputs (Figure 2d). The player (manager)

294 inputs consist of two sliders, setting the cost for two out of the three actions available 3 to farmers

295 in time $\square+1$ : killing animals (presented as the cost of a hunting licence) and scaring animals off

296 their land (presented as the cost of a scaring licence). Management budget allocated to one cost

297 cannot be allocated to the other, and any remaining budget is not rolled over to the next time

298 step. The third action available to farmers (tending crops) cannot be directly4 affected by the 299 manager (player), so no input is available for it .

300 The game progresses to the next time step $\square+1$ once the player confirms their choice of cost 301 inputs. At this point (1), the user, resource and observation models are run using the updated 302 action costs set by the player, (2) selected environment state data are stored in the database (See 303 3.2.3 below), and (3) trajectory, landscape and action plots are updated, and budget allocation is 304 reset. The current implementation of A\&F continues for a maximum of 20 time steps (following 305 the initial five) at which point the game session is ended and the player is presented with a 306 scoreboard. If the resource population reaches extinction in any given time step, the game 307 session is also terminated.

\section{$308 \quad 3.2 .3 \quad$ Game objective, scores and scoreboard}

309 The game objective presented to the player is "to maintain the number of animals and overall 310 agricultural yield of your choice." Thus, the player is asked to make management decisions

3 A\&F currently focuses only on hunting animals, scaring animals or tending crops as available actions to farmers; this may be expanded in the future to other actions available in GMSE.

4 It can be affected indirectly by setting the cost for the two actions prohibitively high, so that tending crops becomes more likely to be most beneficial to maximising yield (the farmer's goal). 
311 reflecting their preference of animal population and agricultural yield trajectory. At the end of a

312 game session the player is presented with two scores which allows them to assess their

313 performance relative to their own previous game sessions as well as those of other players. The

314 scores are arbitrarily defined to reflect performance in terms of the animal population ("animal

315 score") on the one hand, and overall agricultural yield ("yield score") on the other. Both scores

316 can be interpreted as the mean \% of the initial starting score, which is set at 100\% (see S1 for

317 further details). They are updated and displayed at each time step, and the final scores are

318 displayed on a score board after the final time step is complete, or once the animal population

319 goes extinct. The scoreboard is a top 10 "leaderboard" of scores over all sessions played by all

320 players to date; if the current player's score is not included in the top 10, it is displayed at the

321 bottom of the board with the correct rank relative to other players.

\section{3.2.4 Data collection \& database}

323 Game play data (e.g. session variables, player inputs, environment state variables) are stored in a 324 MySQL relational database. Database structure is outlined in S1. A full list of parameter values 325 stored, and their description, is given in Table S2. This represents only a subset of all GMSE 326 parameters and may be easily extended in the future by adding additional fields to the relevant 327 database table and ensuring the database interface functions append the extra parameters. For any 328 GMSE parameters that are not stored currently, the default GMSE parameter values are used.

\section{$4 \quad$ Example application}

\section{$330 \quad 4.1 \quad$ "Sandbox" for in silico experiments}

331 The combination of the underlying modelling framework, game interface, and the database back332 end, provides a platform to collect data on player interaction with the models in a range of 333 simulated environments. This might include in silico tests of the effect of specific variability in 334 the environment on simulated animal population extinction, or collecting "big data" on player 335 decision-making given a set of (more or less) variable parameters in terms of animal population, 336 observation, or user models. For example, a researcher using the platform may be interested in 
337 testing how human decision-making varies depending on the extent of observed variation in 338 either the ecological (e.g., more or less uncertainty in animal population trajectories) or social

339 (e.g., higher or lower variability in land ownership or sizes of farmer budgets) parts of the 340 modelled system. Data from such experiments may then be combined with debriefing interviews 341 with players to further investigate what may drive such decision-making (e.g., Rakotonarivo, 342 Bell, et al. 2021). Alternatively, by collating large quantities of decision-making data under 343 varying parameter settings, in addition to the outcome of each game session (e.g., animal 344 population extinction and/or trajectories), it may be possible to develop algorithms that can make 345 decisions that are most likely to lead to a desired outcome (e.g. minimising extinction probability 346 while maintaining agricultural yield, or maximising one or the other). While the genetic 347 algorithm for manager decision-making currently implemented in GMSE is effective, it does not 348 currently balance multiple objectives, nor does it necessarily accurately reflect variability in real349 life decision-making processes. Parameterising an alternative algorithm based directly on 350 empirical decision-making data has the potential to address these shortcomings.

\section{$351 \quad 4.2 \quad$ Example scenario \& method}

\section{$352 \quad 4.2 .1 \quad$ Rationale \& methods}

353 Here we illustrate one aspect of this potential by collecting decision-making data from a sample 354 of test players. The main aim was to (1) obtain feedback on the model-game set up, and (2) 355 collect example data to illustrate the potential of the approach, with specific emphasis on how 356 communication of it may be improved in the future. We circulated a link to the game with 357 scenarios configured as detailed below to a sample of 45 contacts working in conservation 358 science and practical conservation and management, covering a range of academic institutions, 359 research institutes, NGOs and government. Contacts were also asked to share the link with any 360 potentially interested contacts. An accompanying covering letter explained this aim, the 361 background to the work, and a request to respond with any feedback. It should be stressed that 362 the data collected here should not be interpreted as comprehensive research on a specific 363 question. It is intended as illustrative of the approach only. 
364 For this proof of concept, we chose to focus on a scenario that systematically varies two 365 parameters, farmer land ownership distribution $\square_{\square}$ and the number of farmers ( $\square$ ). While 366 inequity in land ownership is commonplace and of interest to conservation strategies (e.g., 367 Rakotonarivo, Bell, et al. 2021), the current manager decision-making algorithm implemented in 368 GMSE cannot explicitly account for the extent of such variation. Thus, collecting empirical data 369 on how decisions and resultant population trajectories may be affected by variable land 370 distribution is important.

371 Each new game session is initialised with a random draw of one of three possible values of $\square_{\square}$, 372 representing low, moderate, and high variability in land ownership (resulting landscape patterns 373 illustrated in Figure 3) and one of nine possible values of $\square$, i.e. 4-12 farmers. In addition to this 374 variability, each session also has a small amount of random population mortality $\left(0.05 \leq \square_{\square} \leq\right.$ 375 0.2), sampled from a uniform distribution. All other parameters are kept constant within and 376 between all game sessions. Although the landscape ownership distribution is clearly shown to the 377 player throughout the game (Figure 2), the player is not told explicitly that ownership will vary 378 before a session starts, or what the extent of this variability will be. This was done to ensure that 379 a player would not selectively abort sessions. Other than this scenario-based parameter variation, 380 game play progresses as described above, with the player able to make management decisions 381 (setting costs for farmer actions) over 20 time steps following the initial five. 
(a)

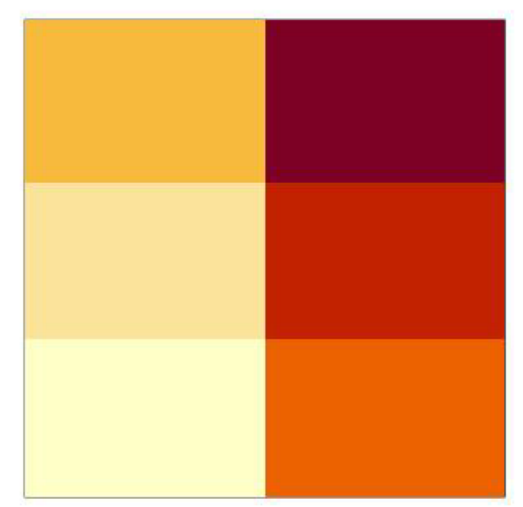

(b)

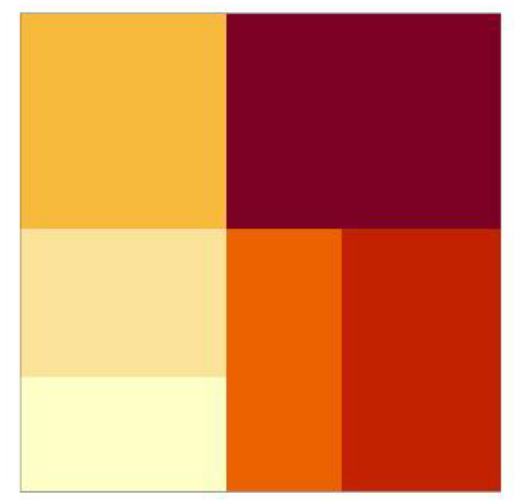

(c)

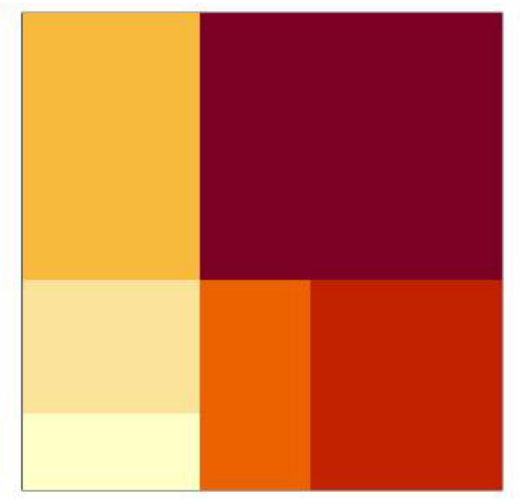

383 Figure 3. Examples of landscape ownership distributions, (a) low variability, $\square_{\square}=0$ (equal distribution),

384 (b) medium variability, $\square_{\square}=0.25$, and (c) high variability, $\square_{\square}=0.5$, here shown for 6 farmer-

385 landowners.

\section{$386 \quad$ 4.2.2 Ethics}

387 The work described here was approved by the University of Stirling's General University Ethics 388 Panel (GUEP), project no. 2519. While the game link is publicly accessible, it was not publicised 389 beyond the direct contacts described above. On accessing the link, players are presented with a 390 series of introductory screens explaining the background and purpose of the game, followed by a 391 digital consent form, with a confirmation tick box. No personally identifiable data are collected 392 or stored, other than a player nickname - the latter is only requested so that scores can be shown 393 in context and compared to other players; however this can be left as a default placeholder, and 394 players explicitly told that this is not expected to be their real name. Player nicknames are 395 replaced by random identifiers prior to further data processing.

\section{$396 \quad \mathbf{4 . 3} \quad$ Illustrative results}

397 Note that the results presented here are intended as illustrative of the model-game approach only, 398 and should be interpreted as such. 
399 Between 21 July 2021 and 19 August 2021, we collated data on 76 play sessions by 28 unique 400 players5; this equated to a total of 1189 decisions (costs set). Sessions lasted 4.5 minutes on 401 average (median 1.6 minutes, range 0.2 - 179.4; the latter maximum duration recorded was an 402 outlier, likely caused by a game session not having been finished and the browser window left 403 open). As per the scenario set up, these sessions were roughly equally distributed between land 404 ownership variability $\square_{\square}(0,0.25$ or $0.5, \mathrm{~N}=21$ [28\%], 32 [42\%], and 23 [30\%], respectively) 405 and number of farmers $\square$ (4-12).

406 The animal population reached extinction in 23 out of the 76 sessions (30.3\%). Extinction 407 probability appeared to be higher at both higher levels of land ownership variability $\left(\square_{\square}=0.25\right.$ 408 and $\square_{\square}=0.5$ ), particularly so at intermediate ( $\square_{\square}=0.25$ ) levels (Figure 4a). Differences in 409 extinction probability with variability in farmer (stakeholder) number was less pronounced 410 (Figure 4b).

411 These extinction probabilities were reflected in the animal population trajectories in each 412 parameter scenario. Figure 5 shows trajectories per level of landownership variability, with cases 413 where the population reached extinction highlighted in red. Both higher levels of variability $414 \quad\left(\square_{\square}=0.25\right.$ and $\square_{\square}=0.5$ ) show fewer cases with rapid increasing trends.

415 Management actions taken by the players (over time, $\square>5$ ) are summarised in Figure 6. It is 416 notable that when land ownership variability was higher ( $\square_{\square}=0.5$ ), chosen costs for hunting 417 licences appeared to be more stable (i.e., less variable), particularly toward the end of playing 418 sessions (Figures 6c vs. 6a-b). It should be noted that this may in part be an artifact of somewhat 419 lower sample size at higher time steps (because in some sessions the population would have gone 420 extinct part way through a session). On average, hunting licence costs also appeared to be set 421 lower overall at higher land ownership variability. By comparison, costs set for scaring licences 422 appeared to more stable over time (Figures 6d-f).

5 Strictly speaking, unique player names. It is possible for the same player to play under multiple different player names. See Discussion for further details. 

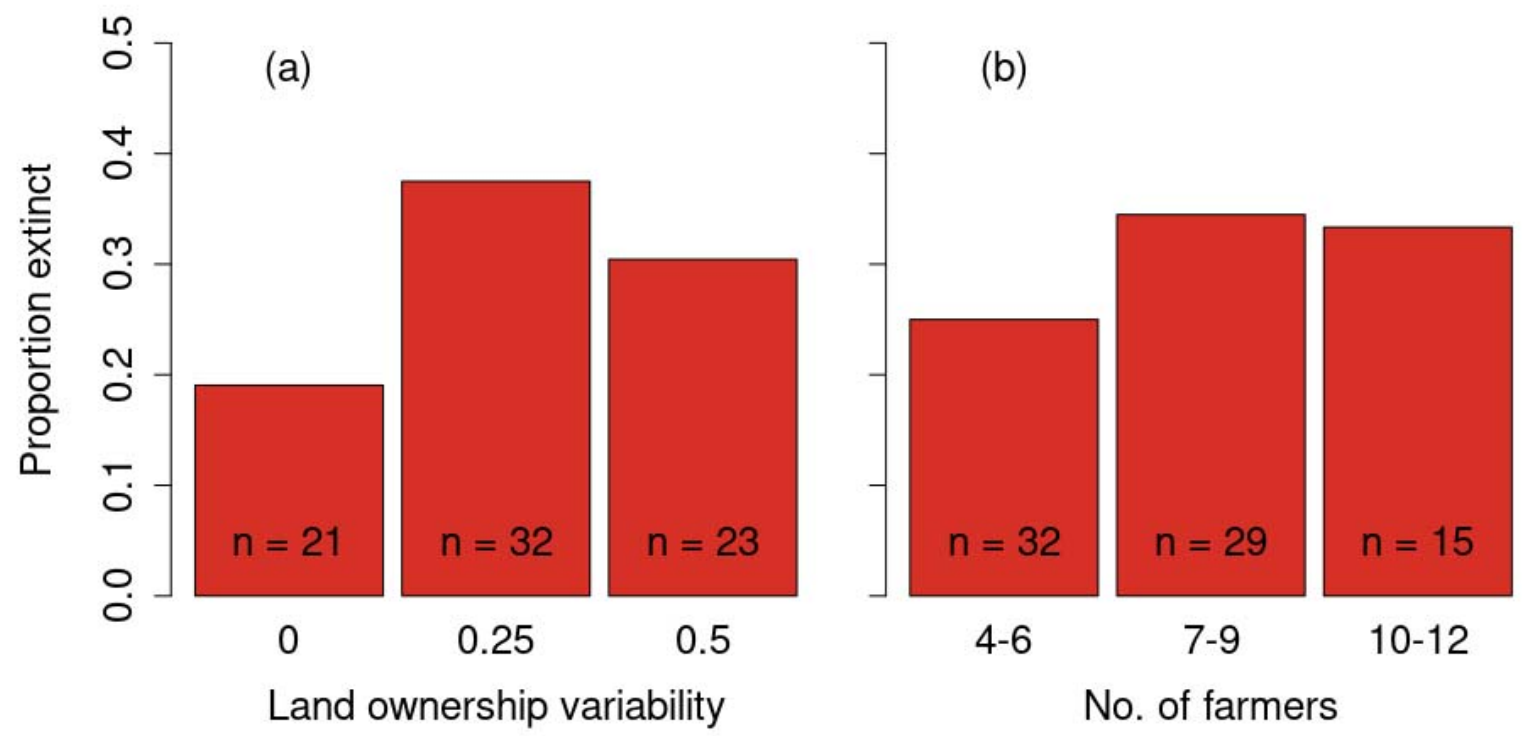

424 Figure 4. Proportion of game sessions where animal population reached extinction, as a function of (a)

425 land ownership variability and (b) the number of farmers (stakeholders) in the game session.

$\mathrm{O}_{\mathrm{v}}=0$

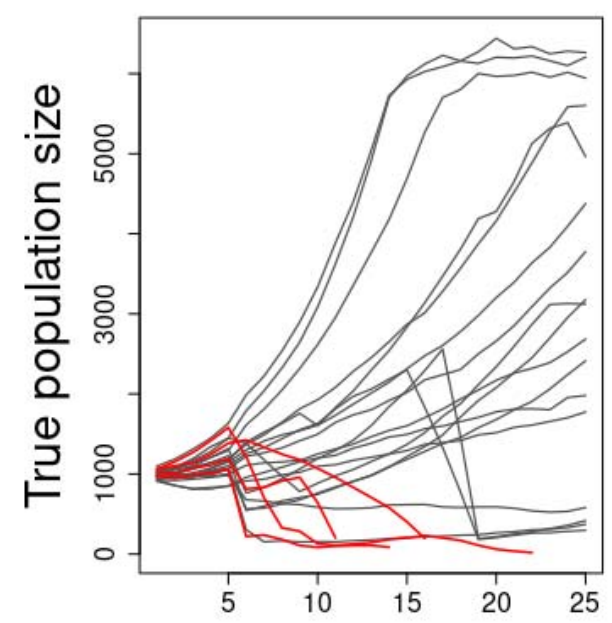

426

$$
\mathrm{O}_{\mathrm{v}}=0.25
$$

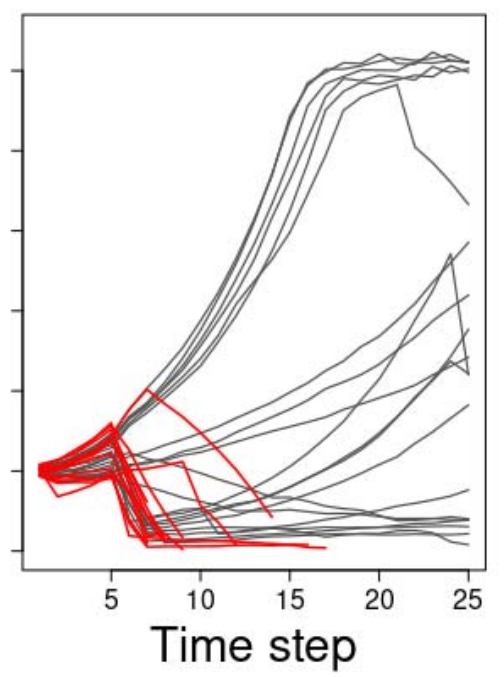

$\mathrm{O}_{\mathrm{v}}=0.5$

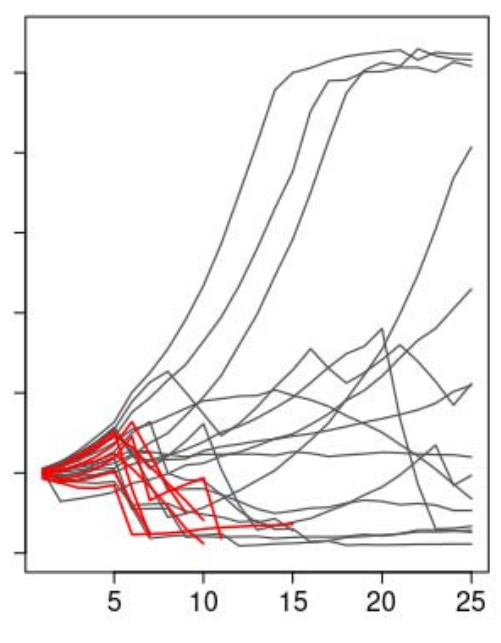

427 Figure 5. Animal population trajectories per game session, split by levels of land ownership variability.

428 Trajectories highlighted in red are sessions where the population reached extinction. 

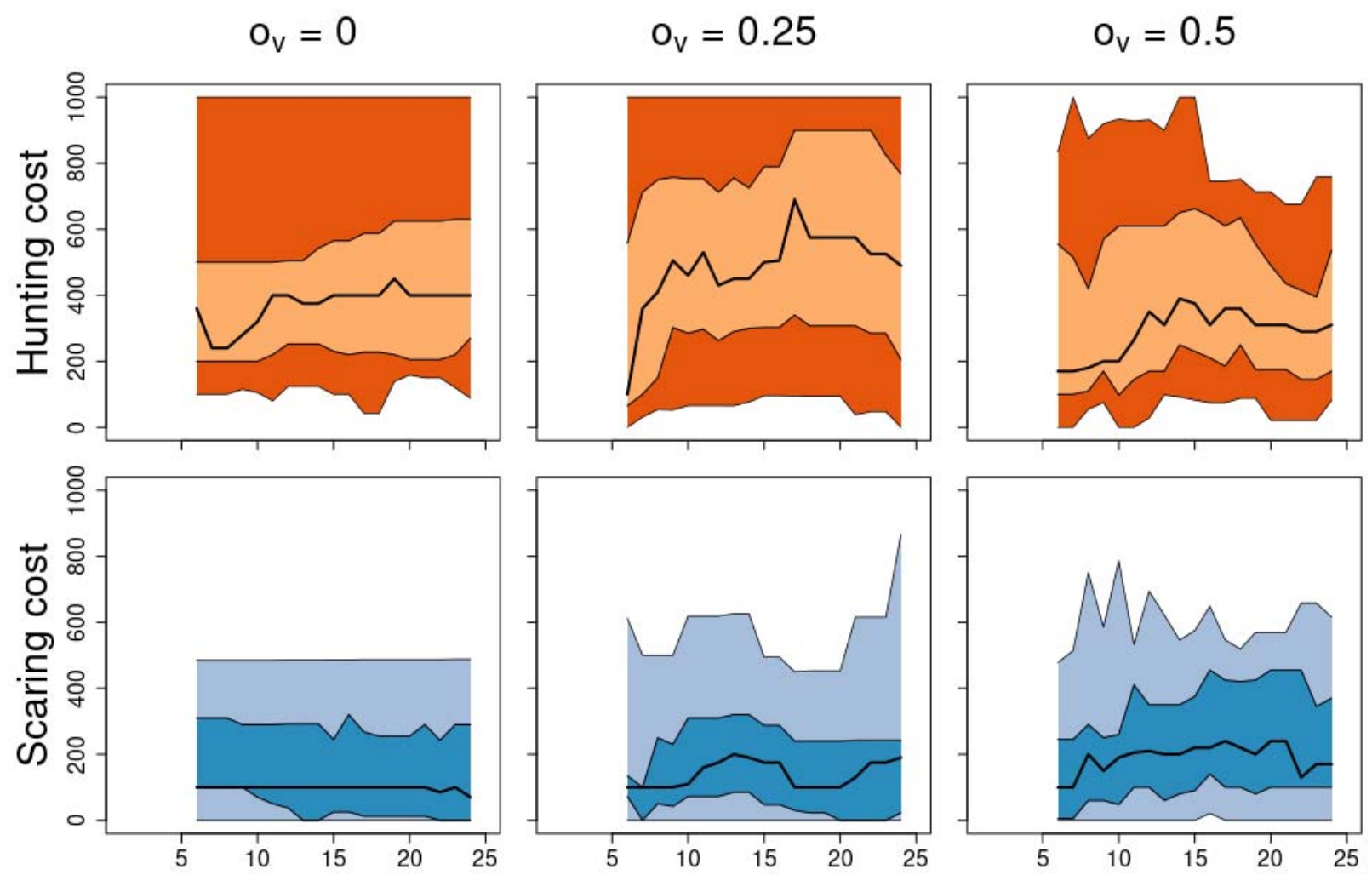

429
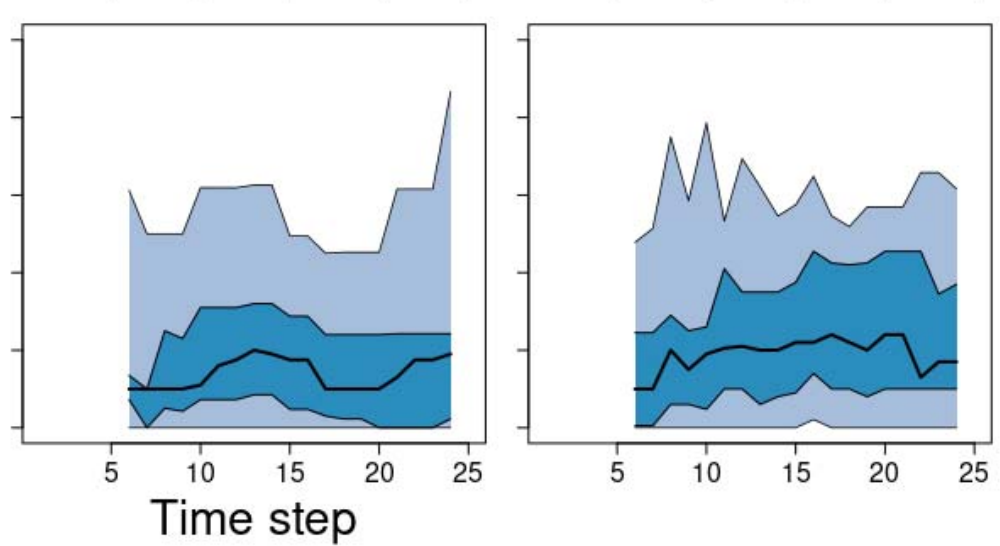

430 Figure 6. Summary of player management actions (costs set for hunting- and scaring licences) over time,

431 per ownership variability scenario. Solid black line is the mean cost per time step, with lighter and darker

432 polygons representing the $25-75 \%$ and $2.5 \%$ and $97.5 \%$ quantiles of the cost distribution per time step.

\section{Discussion}

434 We have here outlined a framework for using an interactive game (A\&F) as an interface to a 435 social-ecological model for natural resource management. The game interface allows players that 436 are not familiar with the underlying model to interact directly and easily with it, with game play 437 decisions directly reflecting parameter settings in the models. We argue that not only does this 438 provide a convenient communication/education tool with respect to both the specific model and 439 models in general, it also provides a tool to both perform in silico experiments on human 440 decision-making in given natural resource management scenarios, as well as collect large 
441 amounts of data that may be used to improve the model parameterisation. It is worth noting that

442 we are here specifically referring to model-games as data-collection tools, as opposed to 443 exclusively as communication- or educational tools.

\section{$4445.1 \quad$ Potential}

445 We illustrated the potential of this approach by presenting data from a small number of trial 446 game play sessions: we showed that subtle variation in farmer land ownership can lead to 447 noticeably different resource population trajectories and manager (player) decision-making 448 patterns. While the data shown here should be taken as illustrative only, it highlights the potential to easily run a range of in silico experiments with direct relevance to real-world 450 questions. For example, uncertainty in the estimation of population numbers (observation 451 uncertainty), and its consequences on decision-making is a perennial topic in conservation 452 management (Nuno, Bunnefeld, and Milner-Gulland 2013). While real-world experiments on 453 this would be extremely challenging and costly, GMSE is a suitable modelling framework in 454 which observation uncertainty can be manipulated, and $\mathrm{A} \& \mathrm{~F}$ provides a platform to run 455 controlled experiments with real-world stakeholders. This approach could extend to many if not 456 all of the 74 parameters currently controllable by users of GMSE, ranging from variability in 457 demography or behaviour of the natural resource, to farmer behaviour or variability, and wider 458 environmental change or stochasticity. The game interface and player interaction would remain 459 the same, with only the underlying architecture and database back end requiring minor 460 adjustment to accommodate the extra parameter variation.

461 In addition to use as an experimental tool, this approach also has great potential for use as a way 462 to source large amounts of decision-making data which may then be used to re-parameterise the 463 underlying models, to better reflect real-world decision making. Given a large enough sample of 464 play sessions with a range of parameter combinations and outcomes, it may be possible to train 465 machine learning algorithms on data collected from this approach, to simulate human decision466 making under a wide range of conditions (Chabris 2017; Duthie et al. 2021). Such algorithms 467 would potentially reflect a range of subtleties of the decision-making process, including 468 balancing multiple objectives in the presence of competing social, financial, and organisational 
469

470

471

472

473

474

475

476

477

478

479

480

481

482

483

484

485

486

487

488

489

490

491

492

493

494

constraints. Algorithms implemented in existing modelling approaches (without reference to empirical data) including GMSE, are limited in how they can represent such "non-rational" decision-making (Constantino et al. 2021; Dobson et al. 2019).

\subsection{Some limitations and potential solutions}

\subsection{1 "The game is unrealistic"}

There are a number of limitations to the model-game approach, particularly in terms of directly using "game-sourced" data to (re)parameterise underlying models. One concern raised by several trial players can be summarised as the game or game play lacking "realism," e.g., lacking aspects or features or real life, or the player's experience of the conservation problem. This could be particularly problematic if any data collected was subsequently used to adjust model parameterisation: if the game world is not seen as sufficiently realistic, it may be argued that player behaviour is not realistic (i.e. perceived lack of realism leading to lack of external validity; (Jackson 2012; Levitt and List 2007), and therefore any reparameterisation would be biased. While a very important point, it is interesting to note that it relates to the underlying model as opposed to the game or the game interface itself. That is, concerns about the lack of "features" or assumptions made are as applicable to any model as they are to the game representation of it, and indeed they are applicable to all models ("all models are wrong," Box 1979). Indeed, this in itself highlights the value of the model-game approach, in that it helps the player to fully understand the model's structure, assumptions, and consequent limitations: particularly given complex social-ecological models, it can be challenging to effectively communicate the full scope of features and limitations (Grimm et al. 2006, 2020). By casting the model as a game, players are put in the center of the modelling process, and any limitations are likely more apparent, more quickly. Recognition of this, particularly by those lacking technical modelling expertise is vital when such models are put to applied use: all models are abstractions of reality and their utility ("some models are useful," Box 1979) depends on careful application and recognition of this. 


\subsection{2 "Humans are biased"}

496 An additional limitation of "gamesourcing" data, either in experimental settings or for 497 parameterising models, is the potential for bias in the audience sample. For example, whether 498 intentionally or unintentionally, it may be that players are sampled from a limited subset. All 499 players may have a single professional background such as conservation science, or the nature of 500 the game (framing) may selectively attract a subset of the public (as in known return bias in 501 questionnaires, e.g. Cheung et al. 2017). As a consequence, the in-game decision-making may 502 not be representative of a wider population of potential players, perhaps by the audience in 503 question being more biased towards conservation rather than social objectives. While this is an 504 important potential issue, we argue that it can be avoided by carefully controlling player 505 recruitment, and subsampling of data collected in different sampling regimes, depending on the 506 research question. This may be achieved, for example, by using game play session codes, 507 separating game sessions for a specific experiment from "open" play sessions (Jones et al. in 508 prep).

509 Similar bias may occur if some players play the game with widely different motivations (e.g., 510 Levitt and List 2007), such as playing to "win" versus deliberately attempting to achieve 511 undesirable outcomes. Indeed, it should be stressed that the scores used in the example 512 implementation presented here are to some extent entirely arbitrary, and the choice of scoring 513 system (including algorithms to calculate them) may inherently bias the decision-making data 514 collected. There are a number of ways in which this issue can be addressed. First, when fully 515 implementing this model-game approach, it will be vital to also collect player data through pre516 or post-game questionnaires, including on for example professional background and social- and 517 ecological attitudes (as in e.g., Rakotonarivo, Bell, et al. 2021; Rakotonarivo, Jones, et al. 2021), 518 which can be used to control for any potential motivational biases in decision-making data. It 519 should be noted that the current example implementation of A\&F allows for anonymous play, 520 and that collection of player personal data would require both further ethical approval as well as 521 additional infrastructure (i.e., unique player names through codes or accounts). Second, it should 522 be stressed that in setting up A\&F, we were careful not to steer players to maximise any specific 
523 objective (See 3.2.3 above; the goal stated in the introductory screens is "your aim is to maintain

524 the number of animals and overall agricultural yield of your choice"). Careful framing of the

525 game objectives (either in open play or in more limited experimental settings) is vital to avoid

526 goal bias (cf. Baynham-Herd et al. 2020).

\section{$527 \quad 5.3 \quad$ Conclusions \& future direction}

528 Provided that the limitations outlined above are taken into account, and the application is

529 carefully considered, we believe that the approach outlined here has great potential to advance

530 both the understanding and capability of complex social-ecological models for natural resource

531 management. As previous work has already shown, games, and in particular videogames,

532 provide a great tool to increase public engagement with quantitative models, and here we

533 highlight how this could be extended to provide effective, flexible and powerful tools for data

534 collection.

535 It is worth stressing that the specific parameterisation of the game presented here, as well as the

536 data collected, is intended as illustrative only. The current game could easily be expanded to give

537 the player more control over the game "world" as is required for a given research question,

538 provided it is supported by the underlying model. More broadly, this proof of concept further

539 supports the case for much wider model-game developments (Duthie et al. 2021). Within the

540 broad theme of natural resource management, more sophisticated games might involve "open

541 worlds" in which a plethora of decisions and strategies are available to players, situated in rich

542 environments that may be affected by (and respond to) decisions in a variety of ways - including

543 potentially other decision-makers in multiplayer settings. Many hugely successful modern

544 commercial videogames (e.g. Red Dead Redemption, MineCraft, Sim City) already provide such

545 highly sophisticated environmental simulations, and the potential for sourcing data on human

546 decision-making (and more broadly) from such virtual environments (or similar custom

547 platforms) is huge. Yet, despite recent developments (Crowley, Silk, and Crowley), this potential

548 remains almost untapped in conservation science and natural resource management. 


\section{Acknowledgements}

550 We thank all the trial players for their time and effort in testing A\&F. Special thanks to Robbie

551 Whytock for comments on the draft manuscript, and to seven of the trial players for providing

552 specific feedback on which much of the Discussion for this paper was based, and which will

553 form a starting point for future improvements of the model-game approach. JM, LTW and NB

554 were funded by EU Horizon 2020 grant agreement no. 679651 (“ConFooBio,” to NB); ABD was

555 funded by a Leverhulme Trust ECF fellowship grant; MN was funded by the Natural

556 Environment Research Council (IAPETUS Doctoral Training Partnership); IJ was supported by

557 a UKRI Future Leaders Fellowship (MR/T019018/1); AB was funded by IAPETUS NPIF 558 allocation, grant code NE/R012253/1 and supported by NERC. 


\section{$\begin{array}{lll}560 & 7 & \text { References }\end{array}$}

561 Addison, Prue F. E., Libby Rumpff, S. Sana Bau, Janet M. Carey, Yung En Chee, Frith C.

562 Jarrad, Marissa F. McBride, and Mark A. Burgman. 2013. "Practical Solutions for Making

563 Models Indispensable in Conservation Decision-Making." Diversity and Distributions 19 (5-6):

564 490-502. https://doi.org/10.1111/ddi.12054.

565 Baynham-Herd, Zachary, Steve Redpath, Nils Bunnefeld, and Aidan Keane. 2020. "Predicting

566 Intervention Priorities for Wildlife Conflicts." Conservation Biology 34 (1): 232-43.

567 https://doi.org/https://doi.org/10.1111/cobi.13372.

568 Box, G. E. P. 1979. "Robustness in the Strategy of Scientific Model Building.” In, edited by

569 ROBERT L. Launer and GRAHAM N. Wilkinson, 201-36. Academic Press.

570 https://doi.org/10.1016/B978-0-12-438150-6.50018-2.

571 Bunnefeld, Nils, Emily Nicholson, and E. J Milner-Gulland. 2015. Decision-Making in

572 Conservation and Natural Resource Management. Cambridge: Cambridge University Press.

573 http://public.eblib.com/choice/publicfullrecord.aspx?p=4866274.

574 Ceballos, Gerardo, Paul R. Ehrlich, Anthony D. Barnosky, Andrés García, Robert M. Pringle, 575 and Todd M. Palmer. 2015. "Accelerated Modern Humaninduced Species Losses: Entering the 576 Sixth Mass Extinction.” Science Advances 1 (5): e1400253.

577 https://doi.org/10.1126/sciadv.1400253.

578 Ceballos, Gerardo, Paul R. Ehrlich, and Rodolfo Dirzo. 2017. "Biological Annihilation via the 579 Ongoing Sixth Mass Extinction Signaled by Vertebrate Population Losses and Declines."

580 Proceedings of the National Academy of Sciences 114 (30): E6089-96.

581 https://doi.org/10.1073/pnas.1704949114.

582 Chabris, Christopher F. 2017. "Six Suggestions for Research on Games in Cognitive Science."

583 Topics in Cognitive Science 9 (2): 497-509. https://doi.org/10.1111/tops.12267.

584 Cheung, Kei Long, Peter M. ten Klooster, Cees Smit, Hein de Vries, and Marcel E. Pieterse.

585 2017. "The Impact of Non-Response Bias Due to Sampling in Public Health Studies: A 
586 Comparison of Voluntary Versus Mandatory Recruitment in a Dutch National Survey on

587 Adolescent Health.” BMC Public Health 17 (1): 276. https://doi.org/10.1186/s12889-017-4189-

5888.

589 Constantino, S. M., M. Schluter, E. U. Weber, and N. Wijermans. 2021. "Cognition and

590 Behavior in Context: A Framework and Theories to Explain Natural Resource Use Decisions in

591 Social-Ecological Systems.” Sustainability Science 16 (5): 1651-71.

592 https://doi.org/10.1007/s11625-021-00989-w.

593 Crowley, Edward J., Matthew J. Silk, and Sarah L. Crowley. "The Educational Value of Virtual 594 Ecologies in Red Dead Redemption 2." People and Nature. https://doi.org/10.1002/pan3.10242.

595 Cusack, Jeremy, A. Duthie, Jeroen Minderman, Isabel Jones, Rocío Pozo, O. Sarobidy

596 Rakotonarivo, Steve Redpath, and Nils Bunnefeld. 2020. "Integrating Conflict, Lobbying, and

597 Compliance to Predict the Sustainability of Natural Resource Use.” Ecology and Society 25 (2).

598 https://doi.org/10.5751/ES-11552-250213.

599 Dobson, Andrew D. M., Emiel de Lange, Aidan Keane, Harriet Ibbett, and E. J. Milner-Gulland.

600 2019. "Integrating Models of Human Behaviour Between the Individual and Population Levels

601 to Inform Conservation Interventions." Philosophical Transactions of the Royal Society B:

602 Biological Sciences 374 (1781): 20180053. https://doi.org/10.1098/rstb.2018.0053.

603 Duthie, A. Bradley, Jeremy J. Cusack, Isabel L. Jones, Jeroen Minderman, Erlend B. Nilsen,

604 Rocío A. Pozo, O. Sarobidy Rakotonarivo, Bram Van Moorter, and Nils Bunnefeld. 2018.

605 “GMSE: An r Package for Generalised Management Strategy Evaluation.” Methods in Ecology

606 and Evolution 9 (12): 2396-2401. https://doi.org/10.1111/2041-210X.13091.

607 Duthie, A. Bradley, Jeroen Minderman, O. Sarobidy Rakotonarivo, Gabriela Ochoa, and Nils

608 Bunnefeld. 2021. "Online Multiplayer Games as Virtual Laboratories for Collecting Data on

609 Social-Ecological Decision Making." Conservation Biology 35 (3): 1051-53.

610 https://doi.org/10.1111/cobi.13633. 
611 Fjaellingsdal, Kristoffer S., and Christian A. Kloeckner. 2019. "Gaming Green: The Educational

612 Potential of Eco - a Digital Simulated Ecosystem." Frontiers in Psychology 10 (December):

613 2846. https://doi.org/10.3389/fpsyg.2019.02846.

614 Fryxell, J. M., C. Packer, K. McCann, E. J. Solberg, and B.-E. Saether. 2010. "Resource

615 Management Cycles and the Sustainability of Harvested Wildlife Populations.” Science 328

616 (5980): 903-6. https://doi.org/10.1126/science.1185802.

617 Garcia, Claude, Anne Dray, and Patrick Waeber. 2016. "Learning Begins When the Game Is

618 over: Using Games to Embrace Complexity in Natural Resources Management." GAIA -

619 Ecological Perspectives for Science and Society 25 (4): 289-91.

620 https://doi.org/10.14512/gaia.25.4.13.

621 Grimm, Volker, Uta Berger, Finn Bastiansen, Sigrunn Eliassen, Vincent Ginot, Jarl Giske, John

622 Goss-Custard, et al. 2006. "A Standard Protocol for Describing Individual-Based and Agent-

623 Based Models.” Ecological Modelling 198 (1): 115-26.

624 https://doi.org/10.1016/j.ecolmodel.2006.04.023.

625 Grimm, Volker, Alice S. A. Johnston, H.-H. Thulke, V. E. Forbes, and P. Thorbek. 2020. "Three

626 Questions to Ask Before Using Model Outputs for Decision Support." Nature Communications

62711 (1): 4959. https://doi.org/10.1038/s41467-020-17785-2.

628 Groeneveld, J., B. Müller, C. M. Buchmann, G. Dressler, C. Guo, N. Hase, F. Hoffmann, et al.

629 2017. "Theoretical Foundations of Human Decision-Making in Agent-Based Land Use Models

630 A Review." Environmental Modelling \& Software 87 (January): 39-48.

631 https://doi.org/10.1016/j.envsoft.2016.10.008.

632 Hamblin, Steven. 2013. "On the Practical Usage of Genetic Algorithms in Ecology and

633 Evolution." Edited by Thomas Hansen. Methods in Ecology and Evolution 4 (2): 184-94.

634 https://doi.org/10.1111/2041-210X.12000. 
635 IPCC. 2021. "Climate Change 2021: The Physical Science Basis. Contribution of Working 636 Group i to the Sixth Assessment Report of the Intergovernmental Panel on Climate Change." 637 https://www.ipcc.ch/report/ar6/wg1/.

638 Jackson, Cecile. 2012. "Internal and External Validity in Experimental Games: A Social Reality 639 Check." The European Journal of Development Research 24 (1): 71-88.

640 https://doi.org/10.1057/ejdr.2011.47.

641 Khatib, Firas, Seth Cooper, Michael D. Tyka, Kefan Xu, Ilya Makedon, Zoran Popović, David 642 Baker, and Foldit Players. 2011. "Algorithm Discovery by Protein Folding Game Players.”

643 Proceedings of the National Academy of Sciences 108 (47): 18949-53.

644 https://doi.org/10.1073/pnas.1115898108.

645 Kolkman, Daniel Antony, Paolo Campo, Tina Balke-Visser, and Nigel Gilbert. 2016. "How to 646 Build Models for Government: Criteria Driving Model Acceptance in Policymaking." Policy 647 Sciences 49 (4): 489-504. https://doi.org/10.1007/s11077-016-9250-4.

648 Levitt, Steven D., and John A. List. 2007. "What Do Laboratory Experiments Measuring Social 649 Preferences Reveal about the Real World?" Journal of Economic Perspectives 21 (2): 153-74. 650 https://doi.org/10.1257/jep.21.2.153.

651 Maxwell, Sean L., Richard A. Fuller, Thomas M. Brooks, and James E. M. Watson. 2016.

652 "Biodiversity: The Ravages of Guns, Nets and Bulldozers." Nature 536 (7615): 143-45.

653 https://doi.org/10.1038/536143a.

654 Meinzen-Dick, Ruth, Rahul Chaturvedi, Laia Domènech, Rucha Ghate, Marco Janssen, Nathan 655 Rollins, and K. Sandeep. 2016. "Games for Groundwater Governance: Field Experiments in 656 Andhra Pradesh, India.” Ecology and Society 21 (3). https://doi.org/10.5751/ES-08416-210338.

657 Milner-Gulland, E. J. 2012. "Interactions Between Human Behaviour and Ecological Systems." 658 Philosophical Transactions of the Royal Society B: Biological Sciences 367 (1586): 270-78.

659 https://doi.org/10.1098/rstb.2011.0175. 
660 Nilsson, L., N. Bunnefeld, J. Minderman, and A. B Duthie. 2021. "Effects of Stakeholder

661 Empowerment on Crane Population and Agricultural Production.” Ecological Modelling 440

662 (January): 109396. https://doi.org/10.1016/j.ecolmodel.2020.109396.

663 Nilsson, L., Nils Bunnefeld, Jens Persson, and Johan Månsson. 2016. "Large Grazing Birds and 664 Agriculturepredicting Field Use of Common Cranes and Implications for Crop Damage

665 Prevention." Agriculture, Ecosystems \& Environment 219 (March): 163-70.

666 https://doi.org/10.1016/j.agee.2015.12.021.

667 Nuno, Ana, Nils Bunnefeld, and E. J. Milner-Gulland. 2013. "Matching Observations and 668 Reality: Using Simulation Models to Improve Monitoring Under Uncertainty in the Serengeti." 669 Journal of Applied Ecology 50 (2): 488498. https://doi.org/10.1111/1365-2664.12051.

670 Orach, Kirill, Andreas Duit, and Maja Schlüter. 2020. "Sustainable Natural Resource

671 Governance Under Interest Group Competition in Policy-Making." Nature Human Behaviour 4 672 (9): 898-909. https://doi.org/10.1038/s41562-020-0885-y.

673 Oultram, Stuart. 2013. "Virtual Plagues and Real-World Pandemics: Reflecting on the Potential 674 for Online Computer Role-Playing Games to Inform Real World Epidemic Research.” Medical 675 Humanities 39 (2): 115-18. https://doi.org/10.1136/medhum-2012-010299.

676 Pérez, María-Esther Del-Moral, and Alba-Patricia Guzmán-Duque. 2014. "CityVille:

677 Collaborative Game Play, Communication and Skill Development in Social Networks.” Journal 678 of New Approaches in Educational Research 3 (1): 11-19. https://doi.org/10.7821/naer.3.1.1167919.

680 Rakotonarivo, O. Sarobidy, Andrew Bell, Katharine Abernethy, Jeroen Minderman, A. Duthie, 681 Steve Redpath, Aidan Keane, et al. 2021. "The Role of Incentive-Based Instruments and Social 682 Equity in Conservation Conflict Interventions.” Ecology and Society 26 (2).

683 https://doi.org/10.5751/ES-12306-260208.

684 Rakotonarivo, O. Sarobidy, Isabel L. Jones, Andrew Bell, Alexander B. Duthie, Jeremy Cusack, 685 Jeroen Minderman, Jessica Hogan, Isla Hodgson, and Nils Bunnefeld. 2021. "Experimental 
686 Evidence for Conservation Conflict Interventions: The Importance of Financial Payments,

687 Community Trust and Equity Attitudes." People and Nature 3 (1): 162-75.

688 https://doi.org/10.1002/pan3.10155.

689 Redpath, Steve M., Aidan Keane, Henrik Andrén, Zachary Baynham-Herd, Nils Bunnefeld, A. 690 Bradley Duthie, Jens Frank, et al. 2018. "Games as Tools to Address Conservation Conflicts."

691 Trends in Ecology \& Evolution 33 (6): 415-26. https://doi.org/10.1016/j.tree.2018.03.005.

692 Redpath, Steve M., John D. C. Linnell, Marco Festa-Bianchet, Luigi Boitani, Nils Bunnefeld, 693 Amy Dickman, R. J. Gutiérrez, et al. 2017. "Don't Forget to Look down Collaborative 694 Approaches to Predator Conservation.” Biological Reviews 92 (4): 2157-63.

695 https://doi.org/https://doi.org/10.1111/brv.12326.

696 Sandbrook, Chris, William M. Adams, and Bruno Monteferri. 2015. "Digital Games and 697 Biodiversity Conservation." Conservation Letters 8 (2): 118-24.

698 https://doi.org/10.1111/conl.12113.

699 Schlüter, M., R. R. J. Mcallister, R. Arlinghaus, N. Bunnefeld, K. Eisenack, F. Hölker, E. J. 700 Milner-Gulland, et al. 2012. "New Horizons for Managing the Environment: A Review of 701 Coupled Social-Ecological Systems Modeling." Natural Resource Modeling 25 (1): 219-72. 702 https://doi.org/10.1111/j.1939-7445.2011.00108.x.

703 Schuwirth, Nele, Florian Borgwardt, Sami Domisch, Martin Friedrichs, Mira Kattwinkel, David 704 Kneis, Mathias Kuemmerlen, Simone D. Langhans, Javier Martínez-López, and Peter 705 Vermeiren. 2019. "How to Make Ecological Models Useful for Environmental Management." 706 Ecological Modelling 411 (November): 108784.

707 https://doi.org/10.1016/j.ecolmodel.2019.108784.

708 Schwarz, Nina, Gunnar Dressler, Karin Frank, Wander Jager, Marco Janssen, Birgit Müller, 709 Maja Schlüter, Nanda Wijermans, and Jürgen Groeneveld. 2020. "Formalising Theories of 710 Human Decision-Making for Agent-Based Modelling of Social-Ecological Systems: Practical 711 Lessons Learned and Ways Forward." Socio-Environmental Systems Modelling 2 (December): 712 16340-40. https://doi.org/10.18174/sesmo.2020a16340. 
713 Sipper, Moshe, and Jason H. Moore. 2020. “Gamorithm.” IEEE Transactions on Games 12 (1):

714 115-18. https://doi.org/10.1109/TG.2018.2867743.

715 Sullivan, Devin P., Casper F. Winsnes, Lovisa Akesson, Martin Hjelmare, Mikaela Wiking, 716 Rutger Schutten, Linzi Campbell, et al. 2018. "Deep Learning Is Combined with Massive-Scale

717 Citizen Science to Improve Large-Scale Image Classification.” Nature Biotechnology 36 (9):

718 820-+. https://doi.org/10.1038/nbt.4225.

719 Tan, Cedric Kai Wei, Jiin Woei Lee, Adeline Hii, Yen Yi Loo, Ahimsa Campos-Arceiz, and 720 David W. Macdonald. 2018. “The Effect of Using Games in Teaching Conservation.” PeerJ 6 721 (April): e4509. https://doi.org/10.7717/peerj.4509.

722 van den Bergh, Jarrett, Ved Chirayath, Alan Li, Juan L. Torres-Pérez, and Michal Segal-

723 Rozenhaimer. 2021. "NeMO-Net Gamifying 3d Labeling of Multi-Modal Reference Datasets to

724 Support Automated Marine Habitat Mapping." Frontiers in Marine Science 8:645408.

725 https://doi.org/10.3389/fmars.2021.645408.

726 Villamor, Grace, and Biola Badmos. 2016. “Grazing Game: A Learning Tool for Adaptive 727 Management in Response to Climate Variability in Semiarid Areas of Ghana.” Ecology and 728 Society 21 (1). https://doi.org/10.5751/ES-08139-210139.

729 Will, Meike, Gunnar Dressler, David Kreuer, Hans-Hermann Thulke, Adrienne Grêt-Regamey, 730 and Birgit Müller. 2021. "How to Make Socio-Environmental Modelling More Useful to Support 731 Policy and Management?" People and Nature 3 (3): 560-72. https://doi.org/10.1002/pan3.10207.

732 Wilting, Harry C., Aafke M. Schipper, Michel Bakkenes, Johan R. Meijer, and Mark A. J. 733 Huijbregts. 2017. "Quantifying Biodiversity Losses Due to Human Consumption: A Global734 Scale Footprint Analysis.” Environmental Science \& Technology 51 (6): 3298-3306.

735 https://doi.org/10.1021/acs.est.6b05296.

736 Zasada, Ingo, Annette Piorr, Paula Novo, Anastasio J. Villanueva, and István Valánszki. 2017.

737 "What Do We Know about Decision Support Systems for Landscape and Environmental 
bioRxiv preprint doi: https://doi.org/10.1101/2021.09.23.461497; this version posted September 26, 2021. The copyright holder for this preprint (which was not certified by peer review) is the author/funder, who has granted bioRxiv a license to display the preprint in perpetuity. It is made available under aCC-BY-NC-ND 4.0 International license.

738 Management? A Review and Expert Survey Within EU Research Projects.” Environmental

739 Modelling \& Software 98 (December): 63-74. https://doi.org/10.1016/j.envsoft.2017.09.012. 